\title{
La familia es la voz del paciente en la interacción con la enfermera
}

\author{
Family as the Patient's Voice in Nurse-Patient Interaction \\ A família como a voz do paciente na interação enfermeira-paciente
}

\begin{abstract}
Como citar este artículo:
Anduquia Vásquez Paula Andrea, Ramírez Barrientos Adriana, Lopera Betancur Martha Adiela, Forero Pulido Constanza, Córdoba Pérez Luisa Fernanda. La familia es la voz del paciente en la interacción con la enfermera. Revista Cuidarte. 2020;11(3):e1015. http://dx.doi.org/10.15649/cuidarte.1015
\end{abstract}

Revista Cuidarte

Rev Cuid. 2020; 11(3): e1015

doi) http://dx.doi.org/10.15649/cuidarte.1015

\section{E-ISSN: 2346-3414}

(1) Paula Andrea Anduquia Vásquez (1) Adriana Ramírez Barrientos ${ }^{2}$

(1) Martha Adiela Lopera Betancur ${ }^{3}$

(1) Constanza Forero Pulido ${ }^{4}$

(1) Luisa Fernanda Córdoba Pérez ${ }^{5}$

1 Facultad de Enfermería Universidad de Antioquia Colombia Medellín Grupo de investigación Emergencias y Desastres. E-mail

paula.anduquia@udea.edu. co Autor de Correspondencia.

2 Facultad de Enfermería Universidad de Antioquia Colombia Medellín Grupo de investigación Emergencias y Desastres. E-mail Adriana. ramirez@udea.edu.co

3 Facultad de Enfermería Universidad de Antioquia Colombia Medellín Grupo de investigación Emergencias y Desastres. E-mail martha. lopera@udea.edu.co

4 Enfermera Maestría en Salud Pública Facultad de Enfermería Universidad de Antioquia Colombia Medellín Grupo de investigación Emergencias y Desastres. E-mail constanza.forero@ udea.edu.co

5 Enfermera Grupo de investigación Emergencias y Desastres. E-mail Ifernanda. cordoba@udea.edu.co

\section{Resumen}

Introducción: La familia experimenta un reto importante cuando uno de sus miembros está hospitalizado, pues los obliga a modificar sus dinámicas en términos de funciones y roles. Esta investigación cualitativa se realizó con familiares que acompañaron a uno de sus integrantes durante una experiencia de hospitalización; se consideró familia a quienes compartieran una historia en común con el paciente. Objetivo: Comprender el significado que le da la familia al papel que desempeña con el paciente en la interacción con las enfermeras durante el tiempo de hospitalización. Materiales y Métodos: Se utilizó el enfoque etnográfico. Se aplicaron 10 entrevistas a personas adultas. Se obtuvo información hasta lograr la saturación. Se tuvo en cuenta los criterios de rigor y los principios éticos. Resultados: La familia considera que tienen el derecho de ser la voz del paciente para interactuar con la enfermera, y la asume como una de sus funciones en su rol cuidador, para lograrlo es indispensable que ésta acuda al llamado, atienda, acompañe y brinde al paciente unos cuidados mínimos requeridos, relacionados primordialmente con la esfera física, de tal forma que en esa interacción demuestre su calidad humana, reconozca las necesidades del paciente y base su comunicación en la verdad y la cercanía. La familia espera una enfermera que los reconozca y los eduque, así mismo que les diga la verdad y se muestre cercana. Conclusiones: La familia se constituye en la voz del paciente como derecho connatural filial y en ese sentido, asume el cuidado del paciente con apoyo de las enfermeras, quienes tienen el deber de generar confianza y buen trato.

Palabras clave: Relaciones Familiares; Atención de Enfermería; Pacientes; Retención en el Cuidado.

Recibido: octubre 7 de 2019

Aceptado: mayo 18 de 2020

Publicado: septiembre 1 de 2020 $\square *$ Correspondencia

Paula Andrea Anduquia Vásquez

E-mail: paula.anduquia@udea.edu.co 


\section{Family as the Patient's Voice in Nurse-Patient Interaction}

\section{Abstract}

Introduction: Families experience a major challenge when a next of kin is admitted to hospital as family dynamics are forced to change in terms of duties and roles. Qualitative research has been conducted with family members who supported one of their next of kin during their hospitalization. Family members were defined as those who had shared a common history with patients. Objective: To understand the meaning given by families to the patient's voice role in the interaction with nurses during hospitalization. Materials and Methods: An ethnographic approach was used. Complete information was collected from 10 interviews with adults. Strict criteria and ethical principles were also applied. Results: Families consider that their right is to be the patient's voice to interact with nurses, assuming it as one of their caregiving functions. To this end, nurses must respond to the call, assist, accompany and provide patients with minimal care required, primarily related to physical care, so they can demonstrate their human qualities, recognize patients' needs and communicate on the basis of truth and closeness when interacting with patients. Families expect that nurses would recognize and instruct them, as well as tell the truth and be close to them. Conclusions: Families are the patient's voice as a matter of connatural right, assuming patients' care supported by nurses, who are in charge of building trust and providing good care.

Key words: Family Relations; Nursing Care; Patients; Retention in Care.

\section{A família como a voz do paciente na interação enfermeira-paciente}

\section{Resumo}

Introdução: As famílias experimentam um grande desafio quando um parente próximo é hospitalizado, pois as dinâmicas familiares são forçadas a mudar em termos de deveres e papéis. Esta pesquisa qualitativa foi realizada com membros da família que acompanharam um de seus parentes durante a hospitalização. Os membros da família foram definidos como aqueles que tinham compartilhado uma história comum com os pacientes. Objetivo: Entender o significado dado pelas famílias ao papel da voz do paciente na interação com enfermeiros durante a hospitalização. Materiais e métodos: Uma abordagem etnográfica foi utilizada. Informações completas foram coletadas a partir de 10 entrevistas com adultos. Critérios rígidos e princípios éticos também foram aplicados. Resultados: As famílias consideram que tem o direito de ser a voz do paciente para interagir com enfermeiros, assumindo-a como uma de suas funções de cuidado. Para isso, os enfermeiros devem responder ao chamado, prestar assistência, acompanhar e prover aos pacientes os cuidados mínimos necessários, principalmente relacionados ao cuidado físico, para que possam demonstrar suas qualidades humanas, reconhecer as necessidades dos pacientes e se comunicar com base na verdade e na proximidade ao interagir com os pacientes. As famílias esperam que os enfermeiros os reconheçam e os instruam, assim como que digam a verdade e estejam próximos deles. Conclusões: As famílias são a voz do paciente como uma questão de direito conatural, assumindo os cuidados dos pacientes apoiados por enfermeiros, que são responsáveis por construir confiança e prestar bons cuidados.

Palavras chave: Relações Familiares; Cuidados de Enfermagem; Pacientes; Retenção nos Cuidados. 


\section{Introducción}

El concepto de cuidado ha sido reconocido como la base disciplinar de la enfermería; implica un encuentro de personas con su historia y su vulnerabilidad. Esta relación de ayuda es esencial para lograr el cuidado. Según García y Colaboradores', el cuidado es el elemento que carga de significado la relación enfermera-paciente. Desafortunadamente durante este proceso, pueden presentarse problemas en la relación entre las enfermeras y la familia. En algunas situaciones, la familia encuentra que el paciente no recibe el cuidado de enfermería que espera, el cual califican como negativo especialmente en el encuentro inicial y en el establecimiento de una relación empática². Según afirman García y colaboradores', las enfermeras no se sienten preparadas para tratar las necesidades de las familias y éstas, no sienten que las enfermeras se interesen en ellos y desestiman sus necesidades emocionales; por otra parte, las familias perciben barreras de comunicación y relaciones jerárquicas que dificultan la interacción con las enfermeras.

\author{
La familia encuentra que \\ el paciente no recibe el \\ cuidado de enfermería que \\ espera, el cual califican \\ como negativo.
}

Se encontraron diferentes estudios ${ }^{3,4}$, que se enfocaron en conocer el punto de vista de los familiares y las enfermeras, en los cuales se toman en cuenta sus opiniones y sus apreciaciones respecto al cuidado. Sin embargo, se considera importante comprender los significados que los familiares le otorgan a su papel en el cuidado de los pacientes y de esta manera generar nuevas evidencias, teniendo en cuenta el cambiante contexto social y legislativo que en forma directa o indirecta incide en las prácticas de cuidado.

El referente de familia es considerado en este trabajo como un grupo humano en el que se desarrollan una serie de relaciones y experiencias que forman patrones y en las que se asumen roles, responsabilidades y funciones que conducen a una dinámica propia y particular, determinando su estructura. Por lo general suelen compartir un espacio físico común 5 . En ese sentido, es de resaltar que, como sistema, la familia recibe estímulos y en esa medida da respuesta a los mismos. Ante una condición de enfermedad, esta es comprendida como un estímulo externo que puede afectar a uno de sus miembros y repercute en las demás personas del grupo. Por tanto, cuando la familia enfrenta el proceso de enfermedad de alguno de sus integrantes, sufre su padecimiento y asume nuevas formas de reorganización en cuanto a roles, actividades y responsabilidades con la intención de conservar la "estabilidad" vinculante ${ }^{6}$, lo cual debe ser reconocido, aceptado y promovido por la enfermera como una forma de apoyo durante el afrontamiento de la crisis.

Es por ello que tener un paciente hospitalizado es todo un reto para la familia, pues los obliga a afrontar las demandas y adaptarse a nuevas formas de interacción ${ }_{\mathbf{L}}$ con la cuota adicional de adquirir nuevos recursos para asumir la incertidumbre que el proceso en sí envuelve para ella y el familiar enfermo. La Asociación Profesional de Enfermeras de Ontario ${ }^{7}$, describe que el cuidado de la enfermera debe estar centrado en el paciente y la familia teniendo en cuenta la experiencia de la persona y el papel de la familia en su vida y el que puede desempeñar a la hora de apoyar en el momento de la enfermedad, se toma en cuenta la unidad del individuo y la familia como un sistema que interactúa permanentemente. Por otro lado, Canga y Esandi ${ }^{8}$ mencionan lo importante que es reconocer la familia en el proceso de cuidado y recuperación del paciente. Sin embargo, parece recibir escaso apoyo en las labores de cuidado y poca atención en cuanto a sus demandas y necesidades, por lo que se cae en el error de sobrecargarla, aumentar sus niveles de tensión y menospreciar sus esfuerzos. En esa medida, la familia pasa a convertirse 
en un recurso y no parte del sujeto de cuidado, lo que afecta su papel de cuidadora y al mismo tiempo su proyecto vital familiar.

Para García, et al ${ }^{1}$, la enfermera como parte del equipo de salud, está llamada a promover la participación de la familia en el cuidado del paciente.

En tanto que sería necesario, reconocerla como elemento básico de soporte afectivo y nexo entre el paciente y el mundo exterior. Mencionan además que: "es imprescindible conocer las necesidades que presentan las familias, así como su percepción y vivencia de la situación, sus creencias y sus expectativas para ofrecer unos cuidados individualizados y de calidad".

tener un paciente hospitalizado es todo un reto para la familia, pues los obliga a afrontar las demandas y adaptarse a nuevas formas de interacción.

El objetivo fue comprender el significado que le da la familia al papel que desempeñan con el paciente en la interacción con las enfermeras durante el tiempo de hospitalización.

Los resultados obtenidos permiten mejorar la interacción con la familia facilitando el cuidado del paciente, teniendo en cuenta la voz de los familiares para que el cuidado gire en un mismo sentido o sea en la solución de los problemas del paciente.

Es importante tener en cuenta que, al conocer el papel de la familia en el cuidado del paciente, se pueden hacer propuestas que faciliten la interacción y la confianza, asunto indispensable en el desempeño de la enfermera.

\section{Materiales y métodos}

El método seleccionado fue la investigación cualitativa con enfoque etnográfico, lo que permitió describir y analizar los procesos culturales de la experiencia de los familiares que tuvieron un paciente hospitalizado; para esta mirada se partió del concepto de cultura planteado por Geertz ${ }^{9}$ como "estructuras de significación socialmente establecidas". La etnografía para este autor, es el proceso de "descripción densa" de la cultura y depende de la mirada de los actores mismos que viven los procesos, lo que corresponde a la "mirada Emic".

Como técnicas para la recolección de la información, los investigadores realizaron 10 entrevistas a personas que acompañaron a un familiar durante una experiencia de hospitalización y que accedieron voluntariamente a participar en el estudio. Estas fueron realizadas por los investigadores y se llevaron a cabo en el lugar seleccionado por los participantes. Este material fue grabado y cada entrevista tuvo una duración promedio de 45 minutos, se obtuvo previo consentimiento de los participantes.

El diario de Campo se utilizó para registrar, durante los encuentros con los participantes, los aspectos que se consideraron importantes, comentarios, aclaraciones y demás asuntos que ayudaron a la comprensión del fenómeno.

La selección de los participantes se realizó mediante el muestreo por bola de nieve; el primer participante se captó por contacto social. Los criterios de inclusión fueron: ser mayor de edad, que hubiesen tenido la experiencia de haber sido familiares de un paciente hospitalizado y en capacidad de responder las preguntas. 
Para el análisis de la información como primer paso se realizó la transcripción de cada entrevista en formato Word y mediante una lectura meticulosa se llevó a cabo un proceso de la codificación que permitió caracterizar y clasificar los datos, construir categorías y subcategorías para finalmente definir los mapas conceptuales que facilitaron la escritura del informe final.

En lo que tiene que ver con los criterios de rigor, la información recolectada se transcribió en el menor tiempo posible para conservar la nitidez de la información que los participantes transmitieron y luego se contrastaron con los hallazgos de otras investigaciones. Los resultados se presentaron a enfermeras investigadoras, pares académicos, enfermeras del área asistencial y en eventos académicos.

\section{Criterios Éticos}

Durante la recolección de la información, se garantizó el respeto por la autonomía de los participantes, quienes tenían la posibilidad de retirarse, aunque ninguno lo hizo; así mismo se garantizó el respeto por las ideas, experiencias y relatos. A cada participante se le solicitó el diligenciamiento del consentimiento informado. Se garantizó la confidencialidad y en ningún momento, desde la transcripción del material recolectado, pasando por el análisis hasta la publicación de los resultados se divulgó la identidad de los participantes. El material recolectado está bajo la custodia de la investigadora principal

La investigación tuvo en cuenta lo dispuesto en la Resolución 8430 de $1993^{10}$, según la cual, se califica este estudio como de riesgo mínimo. Esta investigación fue aprobada por el comité de ética en el acta 256 de diciembre 4 de 2018.

No hay conflictos de intereses de los investigadores para la investigación.

\section{Resultados}

\section{Ser la voz del paciente}

La familia asume la voz del paciente porque considera que este no está en condiciones de tomar decisiones y es la familia quien expresa las necesidades del enfermo, por lo tanto, es un derecho que obtienen, adquirido por el conocimiento que poseen de su familiar hospitalizado.

Como mi mamá no era consciente, nosotros tomamos la decisión por ella.

La familia sabe acerca de la enfermedad del paciente sus problemas, deseos, preferencias; sus comportamientos, alteraciones y cuidados; este saber se obtiene por experiencias previas, por lo tanto, espera que la enfermera la tenga en cuenta y le permita tomar decisiones.

Ya con mi papá lo había vivido, entonces ya nosotros teníamos conocimiento de todo.

La familia tiene claro que debe complementar el conocimiento para cumplir con su función, esto lo hace revisando en internet o consultando a personas que les explican los procesos y los guían acerca del cuidado.

Teníamos familia y conocidos que estudiaron, entonces sacábamos copias o con el celular le tomábamos fotos a la historia o a lo que pudiéramos y ya teníamos preguntas para hacer 
La familia entonces adquiere el derecho de ser la voz del paciente, para lograrlo requiere que la enfermera cumpla con unas obligaciones mínimas para con el paciente de acudir, atender y acompañar.

Nos tenían que atender (la enfermera) porque hay una ley, ustedes tienen que exigir.

Sin embargo, estos mínimos no se cumplen por parte de la enfermera, de ahí que la familia debe asumir el cuidado del paciente.

Uno ya sabe que tiene que estar ahí en el hospital, tiene que cumplir con el deber de cuidar la persona, tiene que estar con ella y estar pendiente.

Asumir el cuidado del paciente es para la familia estar pendiente de las necesidades fisiológicas, de la comodidad, e incluso del cumplimiento del horario de los medicamentos.

Yo veía que ya era la hora del medicamento y se demoraban. O que de pronto necesitara ayuda para bajarlo al baño y las llamaba, y que ya voy, ya voy y ellas no aparecían.

En algunos momentos, la familia busca el apoyo de la enfermera, principalmente cuando considera que debe informar algún cambio en el paciente, pero los profesionales no siempre responden a la búsqueda de apoyo, especialmente en lo que se refiere a la comodidad.

Mi mamá tiene ese lado muy hinchado... y decían que no, que muy raro, que ella no tenía nada... es que nosotros la conocemos, ella no es así, a ella algo le pasa.

En relación con la administración de medicamentos, la familia debe estar pendiente de todo el proceso y en este aspecto le es muy difícil apropiarse del cuidado y aunque tiene el conocimiento, la enfermera no atiende sus sugerencias.

Tuvieron que volver con medicamentos fuertes, que no le protegieron los riñones, nosotros siempre dijimos: por favor es que ella tiene antecedentes, colabórenos con los riñones, pero no, a ellos no les importó.

También la familia considera que otra de sus funciones de cuidado es proteger al paciente de complicaciones como caídas y errores; de igual forma, debe identificar los posibles riesgos

La familia entonces adquiere el derecho de ser la voz del paciente. e informar al equipo de salud para prevenir daños.

Si, nos tocaba hacerlo y uno era pendiente, no se podía ni mover uno de ahí porque de pronto se resbalaba él y caía al suelo.

Por otro lado, los participantes manifiestan que la enfermera tiene muchos pacientes a su cargo por lo tanto no es posible lograr que el cuidado sea personalizado y que tenga una visión de cada uno, como un ser especial y único, en consecuencia, es la familia quien debe darle calor humano, asunto que consideran crucial para la recuperación del paciente.

Si ella estaba tan delicada, ¿a quién necesitaba? a la familia, que ellos como enfermeros no se iban a enfocar con ella porque igual era una paciente más, mientras que nosotros éramos los hijos, y se le podía ayudar en la recuperación. 
Otro aspecto que resalta la familia como indispensable es su obligación de recordándole a las enfermeras las condiciones de su paciente, lo que se espera y las situaciones que se presentan durante la hospitalización, por lo tanto, su presencia es una garantía para el paciente.

Entonces se debe quedar hasta el otro día porque a la enfermera y a todo el mundo se le olvidó por qué razón estaba hospitalizada mi mamá.

Para ser la voz del paciente, cuidarlo y darle el amor que requiere, la familia necesita que las enfermeras los apoyen con unos mínimos relacionados con el cuidado físico, la calidad humana y la ética.

\section{Mínimos para el cuidado físico}

Los mínimos que necesita la familia para realizar los cuidados físicos los relacionan con la comodidad, la eliminación, el control de líquidos, la alimentación, los cambios de posición y la higiene; ellos esperan que el personal de enfermería provea el suministro de los insumos indispensables y los acompañen.

Era yo la que los bañaba, porque ellos simplemente me preguntaban ¿qué necesita? Entonces yo les decía pues una toalla o lo que necesitara y ya ellos me facilitaban eso, pero no me acompañaban.

Además, la familia requiere el acompañamiento mediante la presencia de la enfermera_para que pueda observar y determinar de una manera directa, lo que acontece con el paciente y adicionalmente con aquello que la familia tiene

La familia requiere el acompañamiento mediante la presencia de la enfermera. para contar al respecto.

Mirar que esté orinando bien, es mirar que esté haciendo popó bien, que esté comiendo, que por lo menos esté recibiendo lo que necesita, diariamente, que esté hidratada.

Al no obtener los mínimos en el cuidado físico, se sienten cumpliendo sus compromisos en soledad, por lo tanto, perciben una enfermera ausente.

\section{Mínimos en calidad humana}

Para la familia calidad humana es un cuidado con amor, porque si una persona está enferma y la tratan con delicadeza, la escuchan, eso posibilita su recuperación.

\section{La calidad humana, el amor que se le imprime a lo que se hace.}

Calidad humana está relacionada con aspectos de la educación que no se reciben necesariamente en la instrucción universitaria. Para la familia la enfermera tiene conocimientos, pero le falta calidad humana porque la instrucción universitaria no la da.

Yo me he acercado a gentes educadísimas y que no han llegado ni a ir a la escuela, no son instruidas pero son educadas, porque una cosa es ser educado y otra cosa es ser instruido. 
La calidad humana está emparentada con el amor por el trabajo, con la voluntad de hacer lo mejor_en beneficio del paciente y se ve reflejada en el trato que la enfermera les ofrece.

Tranquila mi amor, tranquila mi reina, espérate yo te la vuelvo a canalizar, no te angusties que vamos a encontrar otra.

También es considerada primordial y la comparan con los recursos, pues, aunque estos pueden ser escasos, si hay calidad humana, hay buena atención.

Si un profesional hace su trabajo con amor, con calidad humana, así los recursos de los hospitales no sean los mismos, para mí eso es primordial.

Cuando la familia no encuentra los mínimos en la calidad humana lo consideran como fallas en la atención y sienten que tienen que hacer intentos, muchas veces fallidos, para lograr algún acercamiento con la enfermera.

Nosotros teníamos que ir a buscarlos, ni en la visita, nada; nosotros íbamos y los buscábamos.

\section{Mínimos éticos}

La familia espera que la enfermera, desde la ética cumpla con el respeto por la dignidad del paciente y la de ellos que los trate como seres humanos, se les acerque, los mire, les hable, los escuche; para ellos es una falta de ética cuando las enfermeras hacen lo contrario; además cuando ellas llevan sus problemas personales al trabajo, son los pacientes quienes sufren las consecuencias de ello, porque la familia asume que están jugando con la vida de las personas.

La que tiene ética es la que saluda: cómo estas, cómo seguís, ya te sentís mejor, es como el acercamiento al paciente y a la familia.

La familia plantea que una condición de dignidad es ofrecerle los mismos derechos de cuidado a todos los pacientes, no importando el pronóstico de enfermedad, la aseguradora y menos las influencias de personas cercanas que favorezcan los procesos.

Aunque ella estaba agonizando, la organizaban, la limpiaban, le daban vuelta, le tomaban los signos vitales como a cualquier paciente, la acomodaban, todo, como si ella todavía fuera una persona que se pudiera recuperar.

Además, ellos esperan que la enfermera le de la información necesaria para el cuidado del paciente sin escudarse en interpretación de las normas, especialmente en la parte de las prohibiciones de dar información.

Dizque por la ética profesional no decirle a uno nada, pero eso no es ética a mí me parece que es egoísmo.

Una de las situaciones que la familia ve como indignas y faltas a la ética son el engaño y las mentiras que utilizan las enfermeras para encubrir las fallas en el cuidado. 
Cuando me entregaron la historia, me di cuenta que no le habían ordenado ningún medicamento, fue una mentira de la enfermera, entonces toda la noche mi mamá se aguantó un dolor porque a la otra, a la enfermera no se le ocurrió que había que ordenarle un medicamento.

Ante estas situaciones, la familia comienza a dudar de la información que recibe de la enfermera y cree que miente si no le soluciona una dificultad.

Aquí no vinieron a ponerle nada, porque ese medicamento que muy difícil de conseguir. ¿Acaso estamos en la selva?

También la familia sospecha que hay algo oculto en los cambios que presenta el paciente, durante el tiempo que ellos no están a su lado, suponen errores, descuidos y omisiones.

Yo creo que hasta la dejaron caer porque cuando a nosotros nos la entregaron ella resultó como con unos puntos y cortada la cabeza, preguntamos si era que le habían hecho un examen o algo, y que no, que nunca; entonces me imagino que se les cayó y por evitar problemas o algo nos lo ocultaron.

La familia al no encontrar los mínimos en la ética siente desconfianza_y por lo tanto tiene que estar atento a cualquier falla en el compromiso de respeto a la dignidad humana del paciente y la familia.

\section{Discusión}

La enfermera en la asistencia hospitalaria tiene el enorme compromiso del cuidado del paciente que lleve a su recuperación o a adaptarse a la situación si es del caso; para llegar a esta meta en términos de calidad del cuidado, es necesario que las enfermeras logren ser permeables a lo que sucede con el paciente y su familia, que logre el acercamiento suficiente para comprender cual es el papel que la familia asume y así poder brindarles las herramientas que le permitan la continuidad del cuidado. Por otro lado, es importante que la enfermera pueda empoderar al paciente para que recupere su voz y su autocuidado. cuando la enfermera

Es necesario que las enfermeras logren ser permeables a lo que sucede con el paciente y su familia. comprende lo que le significa a la familia la interacción con ella, puede adecuar su cuidado en el logro de metas para el paciente y evitar la lucha de poderes con la familia.

En el proceso de hospitalización de un paciente, su familia siente que debe asumir su cuidado y de esta manera evitan que el enfermo tenga autonomía, aminorando sus posibilidades y capacidades; para Foucault ${ }^{11}$, el cuidado de sí implica la relación con uno mismo, con los otros y con el mundo. En este sentido le permite crecimiento personal, pues por medio de la preocupación por uno mismo se modifica, se purifica, se transforma o se transfigura. Para este autor, esto es coherente con la interpretación occidental de la preocupación por uno mismo como algo egoísta y bajo la forma de la obligación para con los otros.

De esta manera, cuando la familia del paciente es su voz, lo cuida y protege, pero este acompañamiento no debería llegar hasta la invasión de la autonomía del paciente para que lleve a cabo algunos cuidados por sí mismo.

La familia espera llevar a cabo funciones de cuidado con el paciente, pero esperan de la en- 
fermera acompañamiento y apoyo, sin embargo, ellos encuentran barreras para comunicarse con las enfermeras, pues su intento no es bien tomado por ellas, quienes desprecian el conocimiento que tienen acerca del paciente y su cuidado, esto coincide con los hallazgos de Bautista y compañeros en un estudio en Colombia "Para los familiares informarse a través del personal terapéutico, no siempre se considera fácil"12,13. Por otro, lado la familia espera obtener información y de no lograrla puede llevar a aumentar su estrés por la incertidumbre, según encontró Marrujo-Pérez et $\mathrm{al}^{3}$.

Con relación a los mínimos que espera la familia de la enfermera, es necesario tener en cuenta que en la interacción de la enfermera con el paciente y la familia cada cual tiene expectativas acerca del otro, siendo esta según Gómez y López"todo aquello que las personas esperan recibir por parte de la enfermera durante el acto de cuidado"13. por otro lado, según Salas y Galiano las familias y las enfermeras tienen diferentes percepciones respecto a lo que es importante en el cuidado, siendo prioritario para las familias las conductas sociales mientras para las enfermeras son prioritarios los asuntos técnicos ${ }^{4}$.

En un estudio realizado en Colombia se encontró que la confianza se adquiere en las primeras interacciones de la enfermera con el paciente "la persona debe reconocer a la enfermera como una persona grata, es decir agradable, generadora de gusto y de complacencia en el cuidado"12, aspecto que coincide en este trabajo, pues para la familia el trabajo con amor y el gusto por la labor van de la mano con la capacidad de brindar un cuidado con calidad.

Swanson ${ }^{14}$, advierte que cuidado es una forma de relacionarse con un ser apreciado, hacia el que se siente un compromiso y una responsabilidad personal, para la teórica el cuidado es una actividad humana que implica conocer la realidad del paciente y su familia, también estar presente o "estar ahí"; hacer por el paciente lo que él haría por sí mismo si le fuera posible, considerando que su autonomía debe ser una condición perentoria para su cuidado. Otro requisito del cuidado es permitir, concepto que se comprende como el hecho de facilitar el paso por la experiencia de la enfermedad y la hospitalización, de tal manera que el paciente y la familia puedan darse cuenta de sus capacidades, manifestar sus inquietudes y encontrar espacios para ser escuchados y apoyados en la solución de sus necesidades. El último aspecto importante del cuidado es mantener la esperanza en la recuperación de la salud y en la capacidad que ellos poseen para superar el evento. En este estudio se encontró que la familia no siempre encuentra una enfermera que pueda cumplir con sus expectativas ni con las planteadas por la teórica, por lo tanto, ellos asumen el cuidado con estas características, sin embargo, conservan un paternalismo que minimiza la autonomía del paciente.

Otro aspecto que llama la atención en los resultados es que la enfermera habla poco con la familia, asunto que esta interpreta como una falta de ética, pues siente que ella se escuda en un interpretación equivocada del secreto profesional, al respecto el código deontológico de enfermería ${ }^{15}$, obliga a guardar la información del paciente bajo reserva del secreto profesional, además estipula que la enfermera no debe hacer pronósticos o evaluación con respecto a los diagnósticos, o terapéutica médica. En contraste, este código estipula que el cuidado se da a partir de la comunicación y relación interpersonal humanizada entre la enfermera y el ser humano, sujeto de cuidado, la familia o grupo social, en las distintas etapas de la vida, situación de salud y del entorno. Es de anotar que la normatividad no limita a la enfermera para ofrecer información acerca de asuntos relacionados con el cuidado.

La familia siente que su compañía es importante para el paciente, de la misma manera la en- 
fermera valora este acompañamiento, debido al aumento de la carga laboral y a las labores colaborativas que la familia pueda realizar para asegurar el cuidado continúo del paciente. Sin embargo, a pesar de que la idea de "colaboración" en el cuidado resulta interesante, hay algunas razones que han facilitado esta situación como lo expresan Canga y Esandi ${ }^{8}$, es la capacidad de reducir costes al gobierno, controlar el gasto público pasando más responsabilidades de cuidado a la familia, lo que puede alterar profundamente las relaciones enfermera-familia y desvirtuar el sentido de implicar a la familia en el proceso de cuidado.

Si bien podría ser cierto que las relaciones de la enfermera con la familia llegan en algunos casos a ser complejas en términos de la comunicación y la dinámica de los servicios asistenciales, Moreno y Colaboradores ${ }^{16}$, expresan que la relación con la familia debe favorecer la escucha y el acompañamiento en la realización de sus funciones de cuidado, y tienen derecho a recibir información clara y oportuna sobre lo que significa ser un cuidador familiar y los conocimientos y las habilidades que deben desarrollar. La familia brinda soporte en la toma de decisiones, acompaña durante la hospitalización y el tratamiento, apoya en las actividades de higiene personal; brinda apoyo emocional y compensa los roles antes desempeñados por su paciente al mismo tiempo que los suyos propios, lo que resulta en un proceso desgastante que puede generar sentimientos de impotencia al no contar con una capacitación y no tener una idea clara del proceso de la enfermedad y de lo que tiene que hacer. También Bautista y colaboradore ${ }^{17}$ encontraron que los familiares resaltan la importancia que la enfermera realice un cuidado humanizado con características como mostrar empatía, trato cordial, compañía y explicación en la realización de los procedimientos, confianza, tranquilidad, y escucha activa, lo cual permite que la familia aumente el grado de satisfacción.

Es indispensable que la enfermera tenga en cuenta que la familia es importante para el paciente, lo mismo que el paciente es importante para su familia. Lo cual coincide con los hallazgos de este estudio.

Por lo anterior, si la familia recibe escaso apoyo y atención, se sobrecarga y esto desvirtúa el fundamento de involucrar a la familia en el proceso de cuidado; es indispensable que la enfermera tenga en cuenta que la familia es importante para el paciente, lo mismo que el paciente es importante para su familia, pero no debe ser tomado como un recurso para aliviar la carga de trabajo de las enfermeras, lo que coincide con los hallazgos de Fernández y colaboradores ${ }^{18}$.

\section{Conclusiones}

El papel fundamental de la familia en el hospital, es ser la voz del paciente ante el cuidado de la enfermera, comprendido como un derecho que naturalmente otorga el vínculo filial. El cuidado del paciente incluye la comodidad, estar pendiente, proteger de los riesgos y darle amor. La familia asume el cuidado del paciente con apoyo de las enfermeras en unos mínimos en lo referente a atender, acudir y acompañar. Escuchar la voz de la familia permite a la enfermera comprender el papel que la familia asume en el cuidado del paciente y así poder realizar acciones educativas y de apoyo que permitan garantizar el cuidado del paciente.

\section{Recomendaciones}

La enfermera debe escuchar a la familia para que su plan de cuidados se haga a la luz de la realidad del paciente. Es importante que las instituciones educativas y asistenciales desarrollen programas educativos dirigidos a la enfermera y la familia para que le den valor al papel de la familia en el cuidado conservando la autonomía del paciente. 
Conflictos de interes: las autoras manifiestan no tener conflictos de interes.

\section{Referencias}

1. García Ortega D, García Graus N, Martínez Martín ML. La relación terapéutica con la familia del paciente crítico. Metas Enferm mar 2016; 19(2): 49-59.

2. Jahuancama-Villagaray $\mathbf{O}$, Espinoza-Moreno T. Relación terapéutica entre enfermera y familia basada en la teoría de Joyce Travelbee, en un hospital de Lima. Health Care \& Global Health.[Internet] 2017[acceso 27 abril 2020] ;1(1):31-35. doi:

http://dx.doi.org/10.22258/hgh.2017.8

3. Marrujo-Pérez KJ, et al. El significado de la hospitalización desde la mirada del cuidador: estudio cualitativo. Enferm Clin. 2016. http://dx.doi.org/10.1016/j.enfcli.2016.01.001

4. Salas Saavedra B, Galiano Galvez M. Percepción de enfermeras y familiares de pacientes sobre conductas de cuidado importantes. Cienc. enferm. [Internet]. 2017 Abr [citado 2020 Abr 27] ; 23( 1 ): 35-44 http://dx.doi.org/10.4067/S0717-95532017000100035

5. Martínez J., Rodríguez G., Diaz A., Reyes M. Nociones y concepciones de parentalidad y familia. Scielo, [Internet]. 2018 [citado 2020 Mayo 04]; 22 (1). Disponible en: doi; https://doi.org/10.1590/2177-9465-ean-2017-0148

6. Waldow Vera Regina. Cuidado humano: la vulnerabilidad del ser enfermo y su dimensión de trascendencia. Index Enferm [Internet]. 2014 Dic [citado 2020 Mayo 09] ; 23( 4 ): 234-238.. http://dx.doi.org/10.4321/S1132-12962014000300009

7. Asociación Profesional de Enfermeras de Ontario (RNAO). Cuidados centrados en la persona y familia [Internet] 2015 [acceso 4 febrero 2020]. Disponible en: https://rnao.ca/sites/ rnao-ca/files/bpg/translations/Person_Family_Ctrd_Care_-_2015_-_cuidados_centrados. pdf

8. Canga A., Esandi N. La familia como objeto de cuidado: hacia un enfoque integrador en la atención de enfermería. Anales Sis San Navarra [Internet]. 2016 Ago [citado 2019 Sep 03]; 39 (2):319-322.Disponible en: http://scielo.isciii.es/pdf/asisna/v39n2/16_cartas1.pdf

9. Geertz C. La interpretación de las culturas. Barcelona: Editorial Gedisa S.A; 1997

10. Ministerio de Salud (CO). Resolución 8430 del 4 de octubre de 1993, por la cual se establecen las normas científicas, técnicas y administrativas para la investigación en salud [Internet]. 1993 [citado 15 julio 2014]. Disponible en:

http://www.minsalud.gov.co/Paginas/Normatividad.aspx

11. Foucault M. hermenéutica del sujeto. Madrid: la piqueta; 1987

12. Bautista L, Arias M, Carreño Z. Percepción de los familiares de pacientes críticos hospitalizados respecto a la comunicación y apoyo emocional. Rev Cuid. [Internet]. 2016; 7(2): 12971309. Disponible en: http://dx.doi.org/10.15649/cuidarte.v7i2.330

13. Gómez J, López L. Expectativa de cuidado de enfermería que tienen las personas en diferentes ámbitos de cuidado. Rev. Colomb. Enferm. [Internet]. 2016 abril [citado 2019 Sep 03]; 12(11):49-56. Disponible en: https://doi.org/10.18270/rce.v11i12.1686

14. Swanson K. La enfermería como el cuidado informado para el bienestar de los demás. Journal of Nursing Scholarship.1993 my-jun: 40(3).

15. Colombia. Tribunal nacional ético de enfermería. Ley 911 de octubre 5 de 2004, por la cual se dictan disposiciones en materia de responsabilidad deontológica para el ejercicio de la profesión de enfermería en Colombia; se establece el régimen disciplinario correspondiente y se dictan otras disposiciones. Bogotá; 2004.

16. Moreno MM, Salazar AM, Tejada LM. Experiencia de cuidadores familiares de mujeres con cáncer de mama: una revisión integradora. Aquichan 2018; 18(1): 56-68.

https://doi.org/10.5294/aqui.2018.18.1.6 
17. Bautista LM, Arias MF, Carreño ZO. Percepción de los familiares de pacientes críticos hospitalizados respecto a la comunicación y apoyo emocional. Rev Cuid. 2016; 7(2): 1297-1309.Â http://dx.doi.org/10.15649/cuidarte.v7i2.330

18. Fernández $\mathbf{P}$, Ignacio $M$, Cervantes L., Jiménez $\mathbf{M}$., Medina M., García A. Cuestionario para evaluar la importancia de la familia en los cuidados de enfermería: validación de la versión española (FINC-NA). Anales Sis San Navarra [Internet]. 2015 Abr [citado 2019 Ago 27] ; 38( 1 ): 31-39. https://doi.org/10.4321/S1137-66272015000100004 